Case report

\title{
What can hide behind an "idiopathic" dilated cardiomyopathy?
}

\author{
Laura Țăpoi ${ }^{*}, 1,2$, Carina Ureche ${ }^{1,2}$, Alexandra Clement $^{1,2}$, Ștefan Ailoaei ${ }^{1,2}$, Radu Andy \\ Sascău ${ }^{1,2}$
}

1"Prof. Dr. George I.M. Georgescu" Cardiovascular Diseases Institute, lasi; 2"Grigore T. Popa" University of Medicine and Pharmacy, lasi, Romania

\begin{abstract}
Myocarditis is an infectious-inflammatory disease with viral infections being one of the most common infectious cause. When it is superimposed to an individual genetic background, myocarditis may progress into a chronic heart muscle disorder, most often dilated cardiomyopathy (DCM), with a natural history similar to classic forms of genetic or idiopathic dilated cardiomyopathies. We present the case of a 30 -year-old patient, with a persistent infectious episode in the last 8 weeks, pain and swelling in the large joints. At admission the patient had fever, tachycardia and a grade $2 / 6$ systolic mitral murmur. Laboratory findings revealed inflammatory syndrome, hepatocytolysis syndrome and microalbuminuria. The electrocardiogram (ECG) showed possible right atrial tachycardia. The echocardiography revealed a globally enlarged heart with reduced ejection fraction and diffuse hypokinesia. When discussing the etiology of the DCM, the following were taken into consideration: a tachycardiomyopathy, ischemic etiology, genetic component, autoimmune etiology (elevated anti-Ro titer), and myocarditis. The diagnosis of myocarditis was confirmed by the cardiac magnetic resonance imaging which showed diffuse fibrosis of the interstitial space and an important increase of the extracellular volume. This case is distinguished by a particular immunological panel requiring dynamic monitoring in order to diagnose a possible associated autoimmune pathology.
\end{abstract}

Keywords: dilated cardiomyopathy; myocarditis; heart failure; echocardiography; autoimmune disease.

\section{Introduction}

Myocarditis is an infectious-inflammatory disease and viral infection is one of the most common infectious cause. The most frequently implicated viruses include adenoviruses, coxsackie B virus, cytomegalovirus and human immunodeficiency virus [1]. When it is superimposed to an individual genetic background, myocarditis may progress into a chronic heart muscle disorder, most often dilated cardiomyopathy, with a natural history

Received: June 2020; Accepted after review: August 2020; Published: August 2020.

${ }^{*}$ Corresponding author: Laura Tapoi, Cardiology Department, "Prof. Dr. George I.M. Georgescu" Cardiovascular Diseases Institute, 50 Bvd. Carol I, 700503, Iasi, Romania

Email: laura.tapoi@yahoo.com similar to classic forms of genetic or idiopathic dilated cardiomyopathies. It is still unclear whether in this case the myocarditis is the cause which uncovers myocardial genetic anomalies, or the genetic alterations favor the evolution to end stage myocardial disease.

\section{Case report}

We hereby present the case of a 30-yearold patient, with a normal weight $(\mathrm{BMI}=21$ $\mathrm{kg} / \mathrm{m}^{2}$ ), without significant personal or family medical history, with a 10-pack year history of smoking and occasional alcohol use, who addressed to the family doctor for a persistent infectious episode in the last 8 weeks accompanied by pain and swelling in the large joints. On this occasion, tachycardia was 
documented and he was addressed to our clinic for evaluation.

On arrival to our facility, the patient had fever $\left(38^{\circ} \mathrm{C}\right)$. The physical examination revealed tachycardia, with a grade $2 / 6$ systolic mitral murmur, mild perimalleolar edema and bilateral basal crackling.

The biological work up revealed inflammatory syndrome $\left(\mathrm{WBC}=10,300 / \mathrm{mm}^{3}\right.$, $\mathrm{ESR}=24 \mathrm{~mm} / \mathrm{h}, \quad$ Fibrinogen $=610.7 \mathrm{mg} / \mathrm{dL}$,
$\mathrm{CRP}=41.1 \mathrm{mg} / \mathrm{L})$, hepatocytolysis syndrome ( $A L T=45 \mathrm{U} / \mathrm{L}, \mathrm{AST}=73 \mathrm{U} / \mathrm{L}, \mathrm{GGT}=67 \mathrm{U} / \mathrm{L}$ ), mild hypoproteinemia $(58.1 \mathrm{~g} / \mathrm{L})$ and microalbuminuria.

The ECG showed supraventricular tachycardia 150/min, inverted $P$ waves in V1, suggestive for right atrial origin, positive $P$ waves in inferior leads suggestive for a superior site of origin- possible right atrial tachycardia (Figure 1).

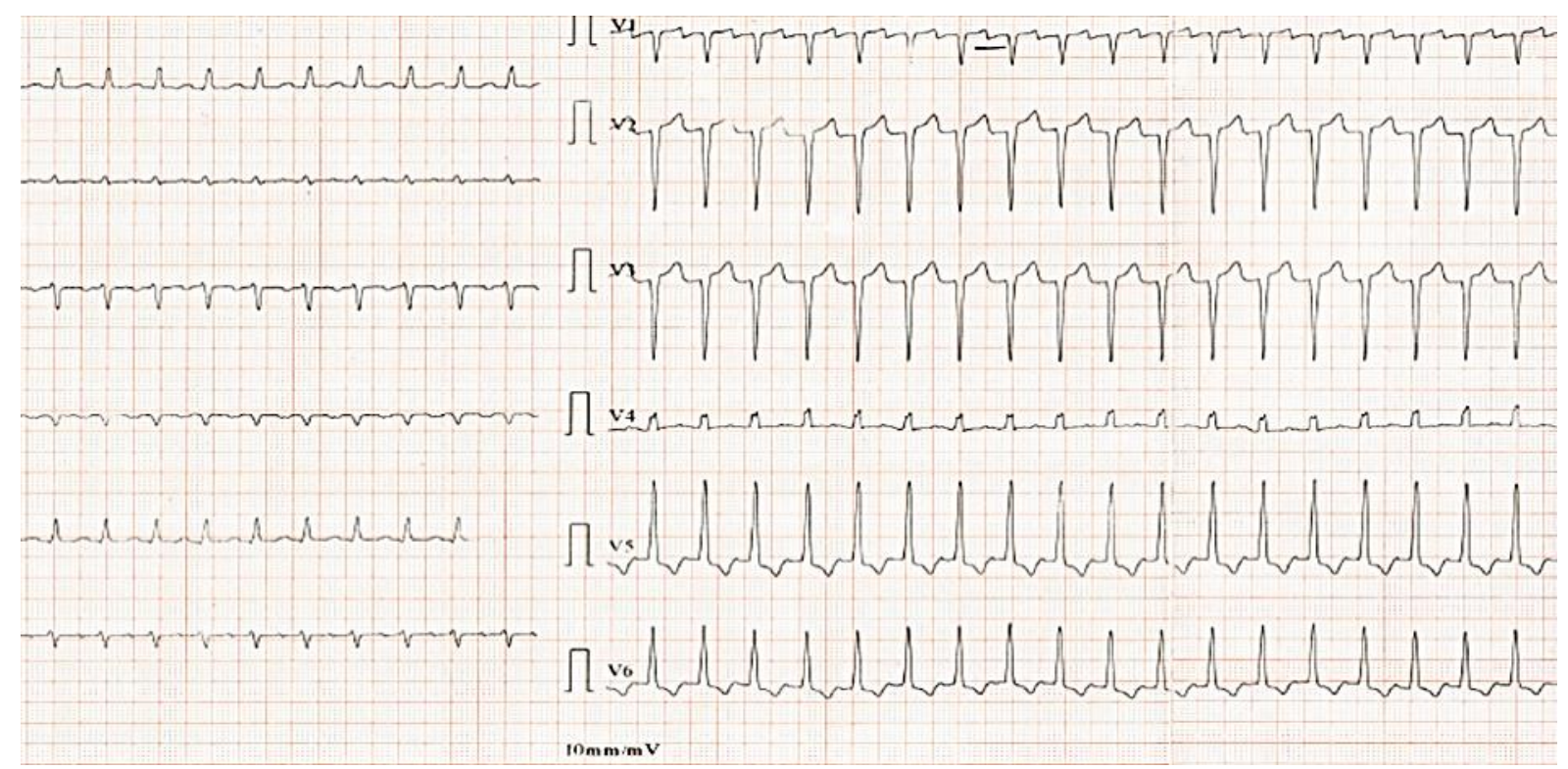

Fig. 1. ECG aspect: Supraventricular tachycardia $150 /$ min, inverted $P$ waves in $V 1$, suggestive for right atrial origin, positive $P$ waves in inferior leads suggestive for a superior site of origin- possible right atrial tachycardia

Transthoracic echocardiography showed a globally enlarged heart, with severe impairment of left ventricular ejection fraction and diffuse hypokinesia (ejection fraction of $20 \%$ by the Simpson biplane method) (Figure 2). There

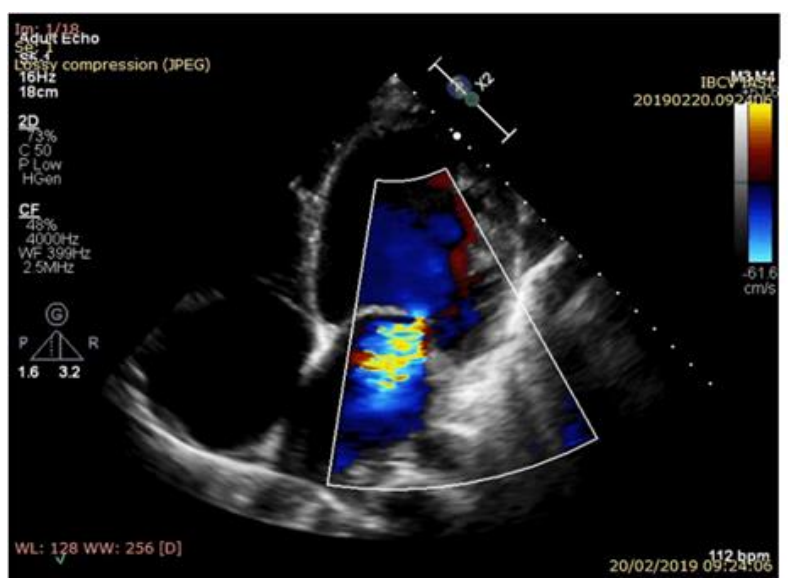

were also moderate mitral and tricuspid regurgitations, with and estimated systolic pulmonary arterial pressure of $30 \mathrm{mmHg}$ and a small posterior pericardial effusion.

Fig. 2. Transthoracic echo: Enlarged heart with severe impairment of LVEF, moderate mitral regurgitation, small pericardial effusion 
Given the initial ECG aspect, a tachycardiomyopathy was taken into consideration. We used beta-blocker (Carvedilol $6.25 \mathrm{mg}$ bid) and Ivabradine $7.5 \mathrm{mg}$ bid, in order to slow down the heart rate and elucidate the ECG aspect. After reaching a more convenient heart rate, the ECG showed sinus rhythm, biphasic $P$ waves with prolonged duration (120ms), which is diagnosis for an advanced interatrial block with left atrial retrograde activation (Bayes syndrome) (Figure 3). As an alternative when the ECG during tachycardia is unclear, adenosine injection and vagal maneuvers may help in clinical diagnosis [2].

At this point in the investigation, we began to search for specific etiologies for the DCM, but only after making sure that we are not facing secondary heart failure etiologies, such as coronary heart disease, hypertensive heart disease or valvular heart disease. Hypertension was excluded and the valvulopathies mentioned above were interpreted as functional. The coronary computed tomography (CT) angiogram excluded an ischemic heart disease (Figure 4).

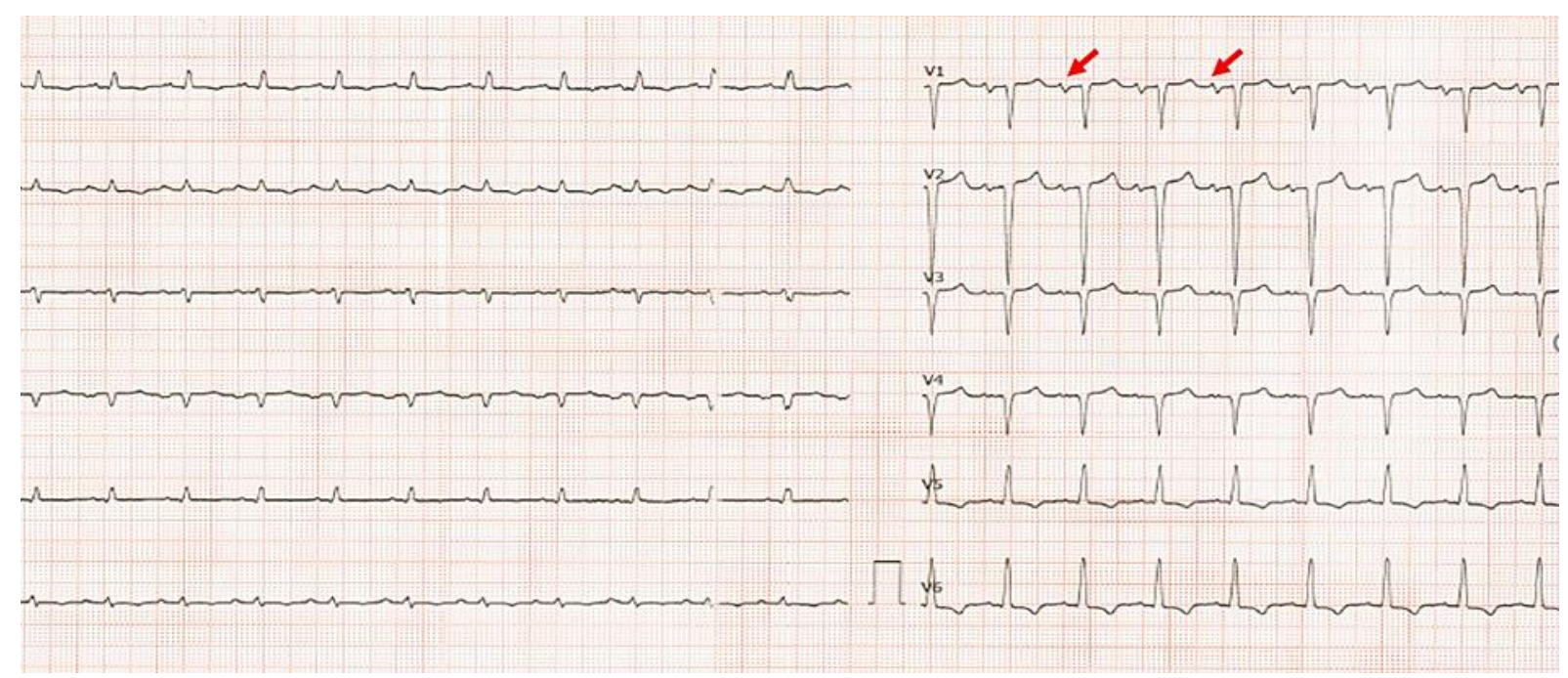

Fig. 3. ECG aspect: Sinus rhythm $80 \mathrm{bpm}$, biphasic $P$ waves with prolonged duration (120 ms), negative T waves in V5, V6, DIl and flattened T waves in DII, aVF

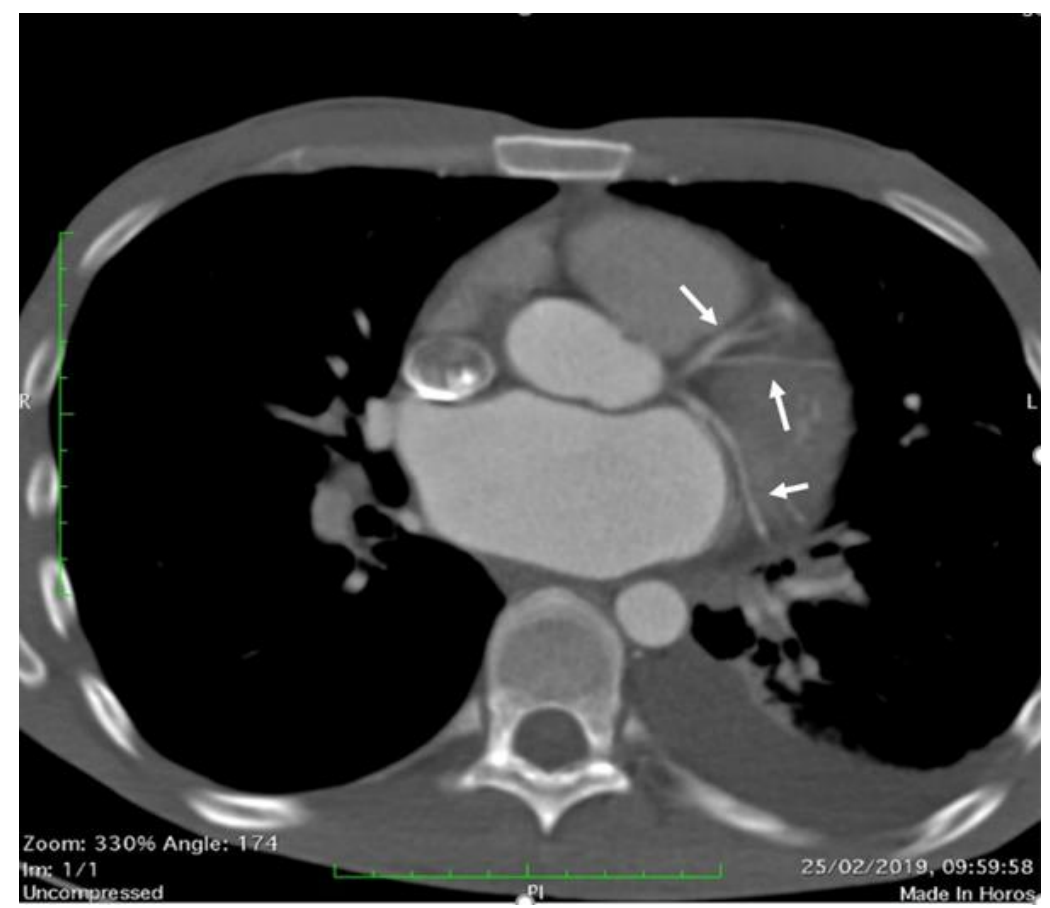

Fig. 4: Coronary CT angiogram: normal epicardial coronary arteries 
When discussing the underlying cause of the DCM, the following were taken into consideration: a genetic etiology, an infection leading to myocarditis, a systemic immunemediated disease, toxic and overload, drugs, as well as a possible endocrine/metabolic etiology [3].

The genetic consultation ruled out a genetic condition. The cultures from the blood, urine, sputum, as well as the pharyngeal exudate were negative. The patient tested negative for acquired immune deficiency syndrome, syphilis and viral hepatitis. Surely, the possibility of a previous viral infection leading to myocarditis remained strong, therefore a cardiac magnetic resonance imaging was performed, showing severe enlargement of the left ventricle

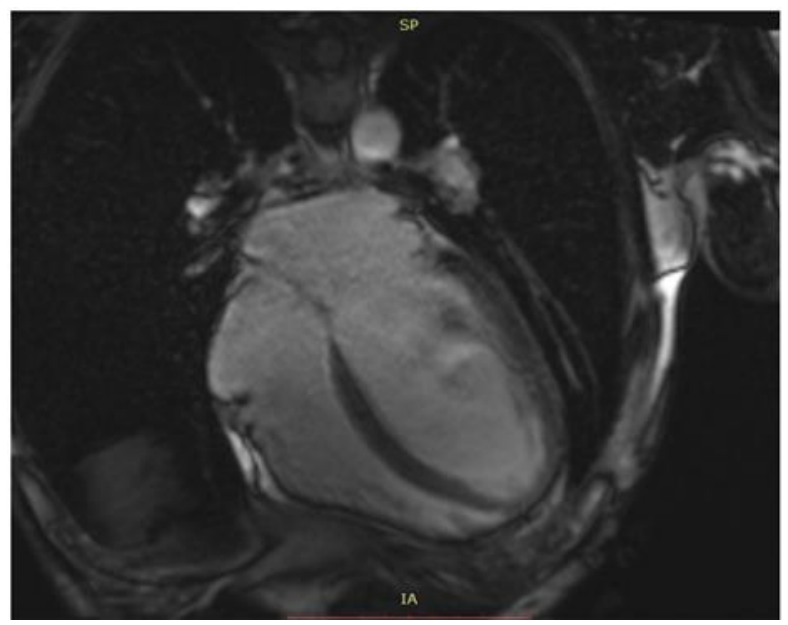

Fig. 5: Cardiac MRI: the presence of diffuse fibrosis of the interstitial space and an important increase of the extracellular volume of the LV (T2Map $42 \mathrm{~ms}$, T1 pre $1280 \mathrm{~ms}$, T1 post $482 \mathrm{~ms}$, ECV 33\%)

Given the AST/ALT =1.6, hypoproteinemia and microalbuminuria, a systemic pathology was suspected and therefore an autoimmune work-up was pursued. The serum antinuclear antibody (ANA) was positive (22.3 $\mathrm{UA} / \mathrm{ml})$ based on the anti-Ro/SSA antibodies (394 $\mathrm{U} / \mathrm{mL}$ ), but at that moment, neither the criteria for Sjogren's syndrome nor for systemic lupus erythematosus (SLE) were fulfilled. Both toxic/overload and drug induced etiologies for dilated cardiomyopathy were excluded. The patient had no clinical/ paraclinical criteria for acromegaly or pheochromocytoma, and the thyroid work up was within normal limits $(\mathrm{TSH}=$ $3.509 \mu \mathrm{Ul} / \mathrm{ml}$, FT4=1.37 ng/dl, Anti-TPO=16.2 $\mathrm{UI} / \mathrm{ml})$. (diastolic diameter $72 \mathrm{~mm}$, systolic diameter 64 $\mathrm{mm})$, moderate to severe systolic dysfunction (LVEF 26\%), with diffuse hypokinesia and impairment in both longitudinal and circumferential strain (-9\% and $-7 \%)$.

There were significant diffuse fibrosis of the interstitial space and an important increase of the extracellular volume, without edema, all of this being suggestive for chronic myocarditis (T2Map: 42ms, T1pre: 1280ms; T1post: 482ms, ECV: $33 \%)$. The right ventricle was slightly dilated with normal systolic function (EDV 203 ml, ESV 102 ml, EF 50\%), but with impairment in both longitudinal and circumferential strain $(-11 \%$ and $-6 \%)$. The left atrium was moderately dilated $(56 / 47 / 51 \mathrm{~mm}$, area $28 \mathrm{~cm}^{2}$ ) (Figure 5).

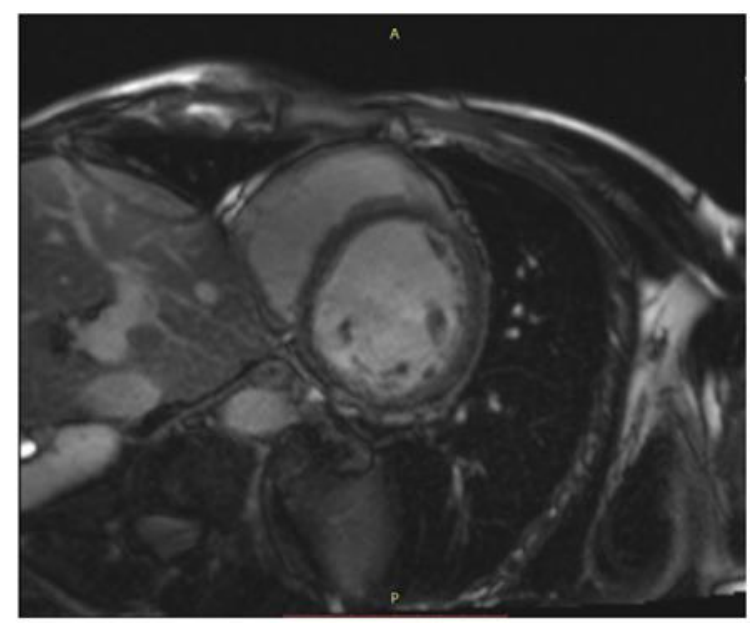

Therefore, the final diagnosis was DCM secondary to chronic myocarditis, and the patient received the treatment for heart failure with reduced ejection fraction, according to the current guidelines [4], with Furosemid $40 \mathrm{mg}$ qd, Spironolactone $25 \mathrm{mg}$ qd, Ramipril $2.5 \mathrm{mg}$ qd, Carvedilol $6.25 \mathrm{mg}$ bid and Ivabradine 7.5 mg bid.

During the first follow-up visit at 3 months, the patient described palpitations and the ECG revealed atrial fibrillation. In this context, we decided to stop the treatment with Ivabradine and initiate antiarrhythmic treatment with Amiodarone, with the restoration and maintenance of sinus rhythm. The reevaluation 
of the left ventricle function showed no significant difference.

At 6 months distance from the initial diagnosis, the patient developed typical discoid lupus lesions localized on the bridge of the nose and the upper cheeks and the dermatological evaluation established the diagnosis of systemic lupus erythematosus (SLE) and treatment with Azathioprine $50 \mathrm{mg}$ bid, Hydroxychloroquine $200 \mathrm{mg}$ bid was initiated.

The echocardiographic reevaluation at 9 months distance from the initial diagnosis showed little improvement of the ejection fraction (ejection fraction of $36 \%$ by the Simpson biplane method). The patient was asymptomatic.

\section{Discussion}

Myocarditis usually manifests in patients without comorbidities and it can be caused by viral, fungal or bacterial infections, drugs, toxins, as well as autoimmune or systemic diseases [5]. Despite these various causes, the etiology remains unclear in a large number of cases and it is said that the viral etiology is the most frequent one, with almost 20 known cardiotropic viruses [6]. Myocarditis is encountered in $\sim 10 \%$ cases of sudden death [7] and it is said that men are more inclined to develop DCM and chronic heart failure [8]. While cardiovascular magnetic resonance should be performed in all patients, endomyocardial biopsy (EMB) is strongly recommended in unstable patients because it offers the possibility to administrate targeted therapy [9].

The presented case highlights a myocarditis which was diagnosed in a young patient directly in its final stage of DCM and it is distinguished by a particular immunological panel, which later on led to the diagnosis of SLE. Myocarditis secondary to SLE has a

\section{References}

1. Cooper L, Knowlton $\mathrm{K}$ Myocarditis in Braunwald's Heart Disease: A Textbook of Cardiovascular Medicine, Philadelphia, PA: Elsevier; 2015:1617-1629. prevalence of about $9 \%$ and usually requires EMB for confirmation. However, because of the focal nature of the disease, the biopsy has a low sensitivity. Moreover, until the present moment, there are no clear cut-offs for the sensitivity or specificity of the available diagnostic modalities in lupus myocarditis [10].

\section{Conclusion}

Thus, when facing a young patient with myocarditis, it is important to maintain a high suspicion for systemic immune-mediated diseases, because a correct diagnosis and a pathogenic treatment can sometimes lead to the improvement of the ejection fraction. Moreover, because of the nonspecific clinical and paraclinical presentation, myocarditis is often a diagnosis of exclusion, and in this scenario, a multimodality imaging approach plays an essential role. Contrast-enhanced cardiovascular magnetic resonance is the most extensive imaging tool for both the diagnosis and prognosis of patients with suspected myocarditis. The Lake Louise Criteria are established diagnostic CMR criteria and include hyperemia, edema and necrosis. The presence of 2 criteria indicate the diagnosis of active myocarditis. In addition, the presence of scar or necrosis play an important role in the prognosis of patients with myocarditis [11].

\section{Consent}

Written informed consent was obtained from the patient for publication of this case report and the accompanying images. A copy of the written consent is available for review by the Editor-in-Chief of this journal.

\section{Competing interests}

The authors declare that they have no competing interests.
2. Brugada J, Katritsis DG, Arbelo E, et al. 2019 ESC Guidelines for the management of patients with supraventricular tachycardia. The Task Force for the management of patients with supraventricular tachycardia of the European 
Society of Cardiology (ESC). Eur Heart J 2020; 41(5):655-720.

3. Seferović PM, Polovina M, Bauersachs J, et al. Heart failure in cardiomyopathies: a position paper from the Heart Failure Association of the European Society of Cardiology. Eur $J$ Heart Fail 2019; 21: 553-576.

4. Ponikowski P, Voors AA, Anker SD, et al. ESC Scientific Document Group, 2016 ESC Guidelines for the diagnosis and treatment of acute and chronic heart failure: The Task Force for the diagnosis and treatment of acute and chronic heart failure of the European Society of Cardiology (ESC). Eur Heart J 2016; 37(27):2129-2200.

5. Frustaci A, Chimenti C. Immunosuppressive therapy in myocarditis. Circ J 2015; 79(1):4-7.

6. Caforio ALP, Pankuweit S, Arbustini E, et al. Current state of knowledge on aetiology, diagnosis, management, and therapy of myocarditis: a position statement of the European Society of Cardiology Working Group on Myocardial and Pericardial Diseases. Eur Heart J 2013; 34(33):2636-2648.

7. Fabre A, Sheppard MN. Sudden adult death syndrome and other non-ischemic causes of sudden cardiac death. Heart 2006; 92:316-320.

8. McNamara DM, Starling RC, Cooper LT, et al. Clinical and demographic predictors of outcomes in recent onset dilated cardiomyopathy: results of the IMAC (Intervention in Myocarditis and Acute Cardiomyopathy)-2 study. J Am Coll Cardiol 2011; 58:1112-1118.

9. Biesbroek PS, Beek AM, Germans T, Niessen HW, van Rossum AC. Diagnosis of myocarditis: Current state and future perspectives. Int $J$ Cardiol 2015; 191:211-219.

10. Wijetunga M, Rockson S. Myocarditis in systemic lupus erythematosus. Am J Med 2002; 113(5):419-423.

11. Friedrich MG, Marcotte F. Cardiac magnetic resonance assessment of myocarditis. Circ Cardiovasc Imaging 2013; 6(5):833-839. 